\title{
New distance estimates to Pulsating RR Lyrae Star DM And.
}

\author{
Adanna FraZIER ${ }^{1, *}$ And ThOMAS O’ KUMA \\ ${ }^{1}$ Lee College, Baytown, Texas, United States. afraier@lee.edu \\ "afraier@lee.edu
}

\begin{abstract}
This is a study of the photometric data of the RR Lyrae star, DM And, in the B, V, sdss$i$, and sdss-z filters. The light curve shapes and estimated period $0.631 \pm 0.011 \mathrm{~d}$ in the B-filter, $0.631 \pm 0.012 \mathrm{~d}$ in the V-filter, $0.631 \pm 0.011 \mathrm{~d}$ in the sdss-i filter, $0.631 \pm$ $0.011 \mathrm{~d}$ in the sdss-z filter, and an average period of $0.6301 \pm 0.011 \mathrm{~d}$ represent that of a typical RRab type RR Lyrae star. DM And's magnitude is $12.175 \pm 0.010$ in B, 11.70 \pm 0.010 in $\mathrm{V}, \mathbf{1 1 . 6 0} \pm \mathbf{0 . 0 3}$ in sdss-i and $\mathbf{1 1 . 4 7 5} \pm \mathbf{0 . 0 4 6}$ in sdss-z, with an average magnitude of $11.738 \pm 0.024$. DM And has a measured distance averaged over all filters of $1647 \pm 42 \mathrm{pc}, 1558 \pm 46 \mathrm{pc}$ in V, $1728 \pm 77 \mathrm{pc}$ in sdss-i, and $1653 \pm 95 \mathrm{pc}$ in sdss-z. These results are comparable to GAIA's measured distance of $1572 \pm 157 \mathrm{pc}$. (수) $\odot \ominus 2021$ Astronomy Theory, Observations and Methods Journal
\end{abstract}

Keywords: Stars: variables: RR Lyrae - stars: variables: Cepheids - stars: distances

https://doi.org/10.32374/atom.2021.2.1

\section{INTRODUCTION}

RR Lyrae pulsating stars are important for the measurement of distance within space (Catelan \& Smith, 2014). The star of interest in this study is DM And which has had a right ascension of 23:32:00.71 and a declination of $+35: 11: 48.90$, according to prior observations (Maintz, 2005). It has a radial velocity of $265 \mathrm{~m} / \mathrm{s}$ (Beers et al., 2000). DM And has a documented period of 0.630387 days (Bramich et al., 2014), which is typical for RRab type stars and a V apparent magnitude of 12.40 (Maintz, 2005).

It is interesting to note that previous investigations have resulted in the discovery of a Blazhko effect displayed by DM And (Skarka, 2014). The Blazhko Effect is "a modulation of the light curve of an RR Lyrae star that has a period much longer than that of the primary pulsation cycle." (Catelan \& Smith,
2014). This type of modulation explained by the Blazhko Effect is not uncommon in RR Lyrae type pulsating stars (Catelan \& Smith, 2014). DM And has a documented metallicity $[\mathrm{Fe} / \mathrm{H}]$ of -2.32 (Bramich et al., 2014). This means the DM And is a metal poor star with very few elements heavier than helium and hydrogen within it.

RR Lyrae type stars (DM And, in this study) have a better period-luminosity-metallicity relationship in the sdss filters, particularly i and $\mathrm{z}$, in comparison to other optical filters (Cáceres \& Catelan, 2008). Since this has not been studied extensively, the data measured in this study is useful and relevant to the period-luminosity-metallicity relationship. Using this, DM And was compared to previously recorded distance measurements by GAIA. 


\section{OBSERVATIONS AND METHODS}

Table 1-4 lists the telescopes and their associated observatories, each of them $0.4 \mathrm{~m}$ telescopes of Las Cumbres Observatory (Brown et al., 2013), used to capture the images used in this study and the number of images captured by each telescope per filter.

Table 1. Telescopes Used B-Filter

\begin{tabular}{cc}
\hline Telescope & Images Captured \\
\hline Haleakala Observatory & 15 \\
Tiede Observatory & 29 \\
McDonald Observatory & 9 \\
\hline
\end{tabular}

Table 2. Telescopes Used V-Filter

\begin{tabular}{cc}
\hline Telescope & Images Captured \\
\hline Haleakala Observatory & 15 \\
Tiede Observatory & 31 \\
McDonald Observatory & 9 \\
\hline
\end{tabular}

Table 3. Telescopes Used sdss-i Filter

\begin{tabular}{cc}
\hline Telescope & Images Captured \\
\hline Haleakala Observatory & 15 \\
Tiede Observatory & 31 \\
McDonald Observatory & 9 \\
\hline
\end{tabular}

The observation period lasted for 12 days from October 6, 2019 to October 18, 2019. Each picture was scheduled to be taken every 4.5 hours through four filters: blue filter (B), visual filter (V), infrared filter (sdss-i) and a deep infrared filter (sdss-z),with different exposure times for each filter. The B filter had an exposure time of 120 seconds, the sdss-z filter had an exposure time of 60 seconds, and the sdss-i and $\mathrm{v}$ filters had an exposure time of 45 seconds.

The Our Solar Siblings (OSS) Pipeline (Fitzgerald, 2018) was used to gather images and prepare them for the user. The OSS Pipeline works with various observatories and automatically processes a full night's worth of images. The OSS Pipeline consists of four parts: The image processing and cleaning
Table 4. Telescopes Used sdss-z Filter

\begin{tabular}{cc}
\hline Telescope & Images Captured \\
\hline Haleakala Observatory & 15 \\
Tiede Observatory & 31 \\
McDonald Observatory & 9 \\
\hline
\end{tabular}

pipeline, the photometry pipeline, the all-sky calibration pipeline, and the catalogue construction pipeline. The image processing and cleaning pipeline makes adjustments to the image so that it is user friendly. The photometry pipeline takes place after the image processing and cleaning pipeline and uses automated photemetry routines on the images captured and sorted into photometry catalogues.

The type of photometry used in this study was the Point Spread Function Extractor, PSFEX (Bertin, 2011). This type of photometry assigns a function to the pixels that make up a star and calculates the total light under the function. The photometry catalogues produced by the OSS Pipeline cleans are ready to be used with Astrosource (Fitzgerald, Gomez, Salimpour, Singleton, \& Wibowo, 2021). Astrosource was used along with Spyder (via Python Anaconda) to analyze the data on DM And's period as well as plotting the graphs and light curves using the images captured by the telescope, which are provided in the results section.

The first step was to identify all the stars (including DM And) in every image that is being studied. Once all the stars common to the imageset were identified, the least variable stars were selected to be compared to the target star, DM And. In order to assure the accuracy of the magnitude measurements for the sdss$\mathrm{z}$ and sdss- $\mathrm{i}$ filter's there were adjustments made to the number of comparison stars used. This will be described in more detail in the discussion section of the paper. From there, the light curves for DM And were constructed and the period was determined.

The following equations listed below were used to find the absolute magnitude from the periodluminosity-metallicity relationships, which were then used to undertake distance measurements. Then the distance and distance error measured by GAIA DR2 (Prusti et al., 2016) was found as well as a reddening or $\mathrm{E}(\mathrm{B}-\mathrm{V})$ value of 0.08 , which was estimated using the NASA/IPAC Infrared Science Archives dust maps (Schlafly \& Finkbeiner, 2011; Schlegel, Finkbeiner, \& 
Davis, 1998).

$$
M_{v}=2.288+0.882 \log Z+0.108(\log Z)^{2}
$$

(Catelan, Pritzl, \& Smith, 2004)

$$
M_{z}=0.839-1.295 \log P+0.211 \log Z
$$

(Cáceres \& Catelan, 2008)

$$
M_{i}=0.908-1.035 \log P+0.220 \log Z
$$

(Cáceres \& Catelan, 2008)

$$
\log Z=[M / H]-1.765
$$

(Cáceres \& Catelan, 2008)

$$
[M / H]=[F e / H]+\log (0.638 f+0.362)
$$

(Cáceres \& Catelan, 2008)

While measuring DM And's period, two period estimation algorithms were used, the minimum distance method and the minimum standard deviation method (presented in Table's 5-8) (Altunin, Caputo, \& Tock, 2020). The minimum distance method and minimum standard deviation method use trialled periods. The distance method uses a repeated process in which distances between two adjacent points on flux versus phase plot of DM And are calculated and summed for each trial period. The standard deviation method sorts fluxes based on their phase into binned intervals of .1 from 0 to 1 and the standard deviation of each interval is taken and summed. Both of these methods were used in Astrosource to calculate the period of DM And.

\section{RESULTS}

DM And has an measured period of $0.631 \pm 0.011$ days along with lightcurve that has a very steep incline and a steady decline, which strongly correlates to RRab type RR Lyrae stars.

Tables 5-8 display the middle magnitude, period measurements (through the distance method and PDM method), amplitude and the errors associated with each of these values for the $\mathrm{B}, \mathrm{V}$, sdss-i, and sdss-z filters.

Table 9 displays the distance measurements and measurement errors for GAIA, the V filter, sdss-i filter, sdss-z filter, and the average of all three filters (Viz).

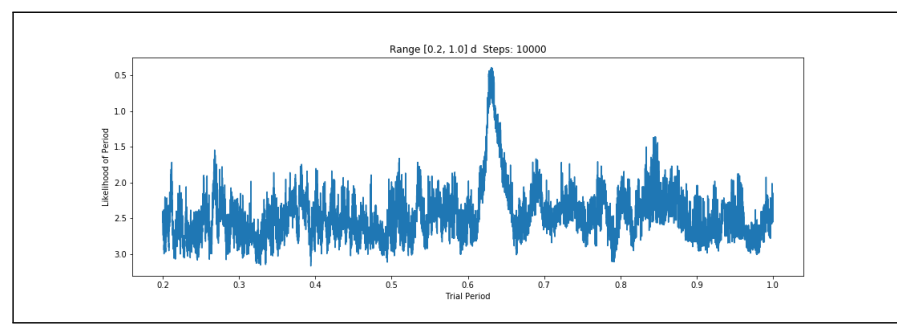

Fig. 1. B-Filter: A likelihood of period versus trial period

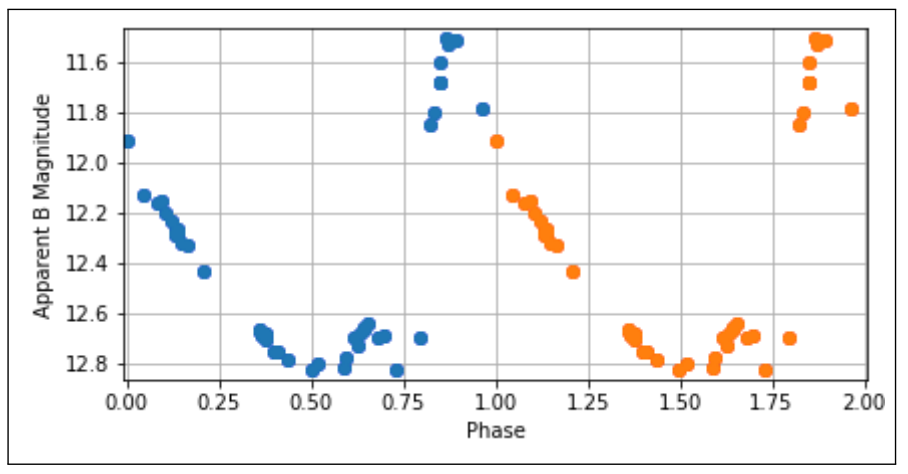

Fig. 2. B-filter: Apparent Magnitude versus Phase

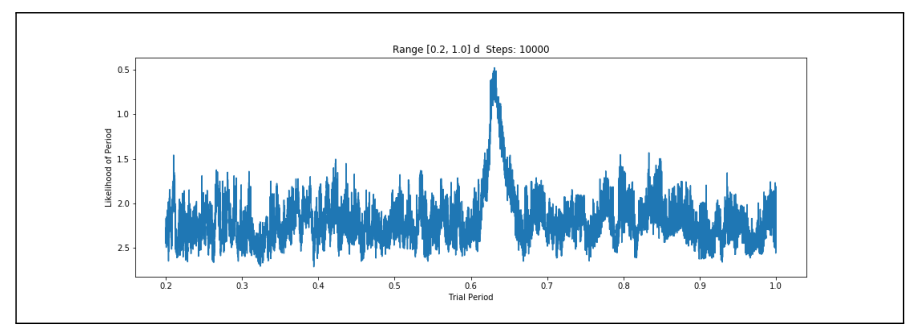

Fig. 3. ip-Filter: A likelihood of period versus trial period

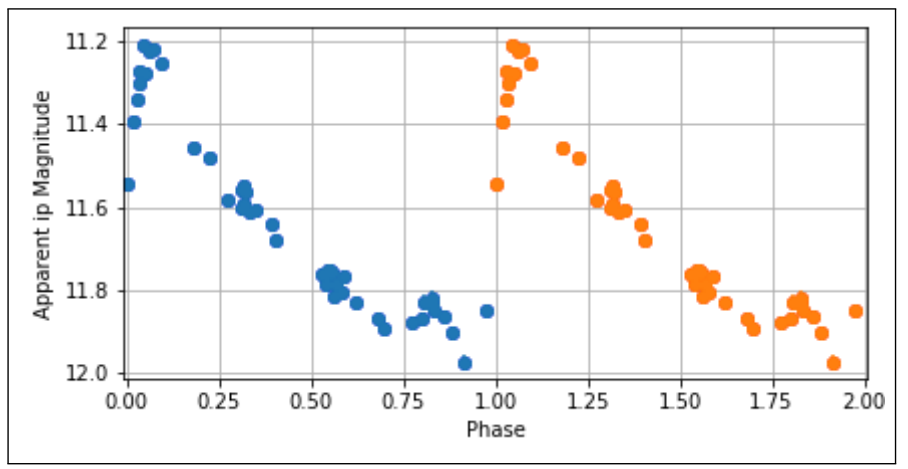

Fig. 4. ip-Filter: Apparent Magnitude versus Phase 


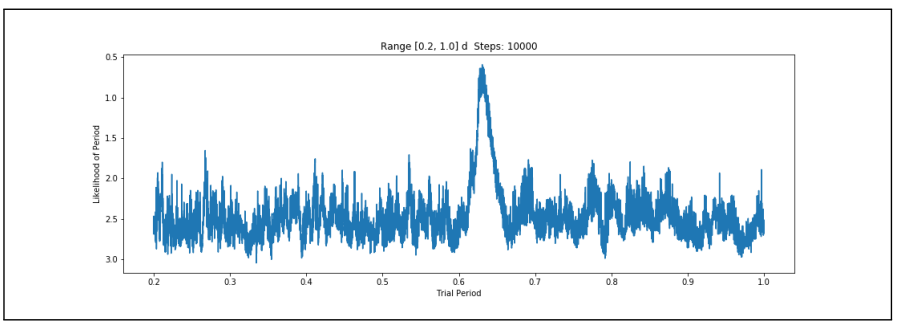

Fig. 5. V-Filter: A likelihood of period versus trial period

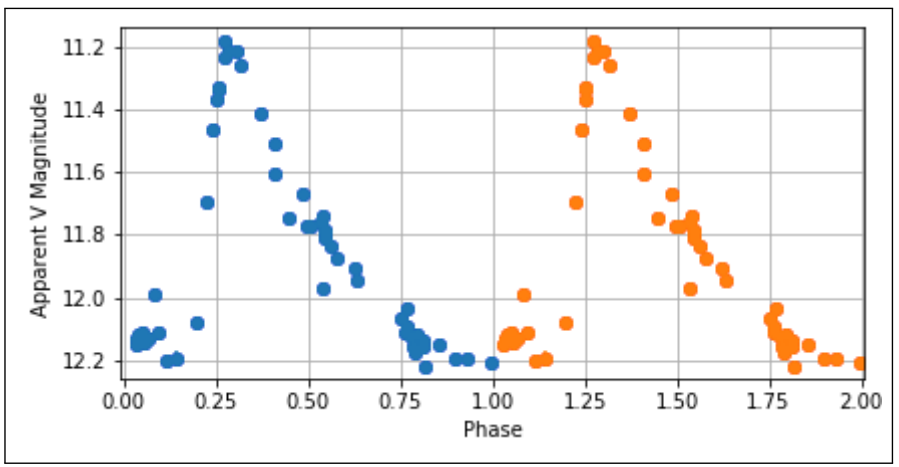

Fig. 6. V-Filter: Apparent Magnitude versus Phase

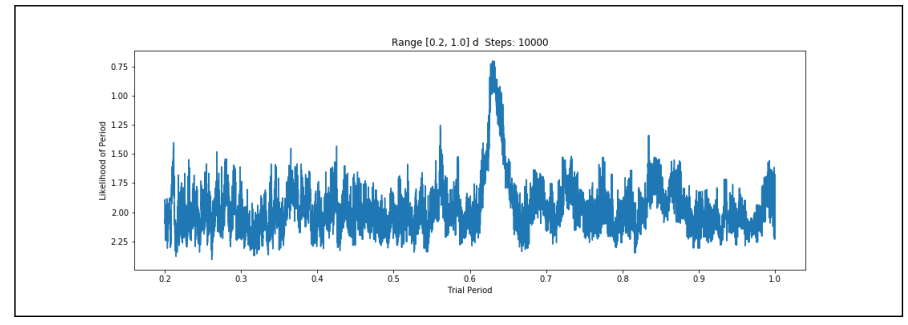

Fig. 7. Zs-Filter: A likelihood of period versus trial period

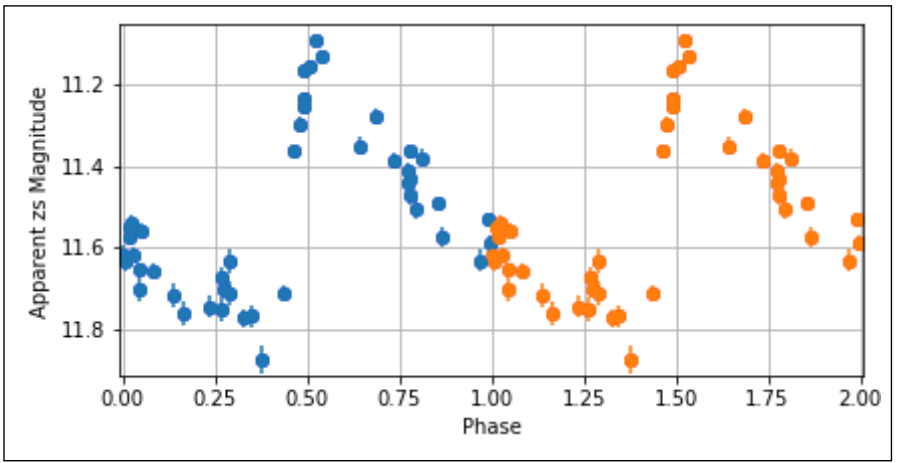

Fig. 8. Zs-Filter: Apparent Magnitude versus Phase
Table 5. Measurements in the B-Filter

\begin{tabular}{ccc}
\hline & B & eror \\
\hline middle mag & 12.175 & 0.010 \\
distance method (days) & 0.6309 & 0.012 \\
PDM Method(days) & 0.6311 & 0.01 \\
amplitude(magnitude) & 0.625 &
\end{tabular}

Table 6. Measurements in the V-Filter

\begin{tabular}{ccc}
\hline & $\mathrm{V}$ & error \\
\hline middle mag & 11.70 & 0.01 \\
distance method(days) & 0.6304 & 0.012 \\
PDM Method(days) & 0.6306 & 0.011 \\
amplitude(magnitude) & 0.5 &
\end{tabular}

Table 7. Measurements in the i-Filter

\begin{tabular}{ccc}
\hline & $\mathrm{i}$ & error \\
\hline middle mag & 11.60 & 0.030 \\
distance method(days) & $0 . .63088$ & 0.012 \\
PDM Method(days) & 0.63088 & 0.01 \\
amplitude(magnitude) & 0.4 & \\
\hline
\end{tabular}

Table 8. Measurements in the zs-Filter

\begin{tabular}{ccc}
\hline & zs & error \\
\hline middle mag & 11.475 & 0.046 \\
distance method(days) & 0.63088 & 0.011 \\
PDM Method(days) & 0.6303 & 0.01 \\
amplitude (magnitude) & 0.375 & \\
\hline
\end{tabular}




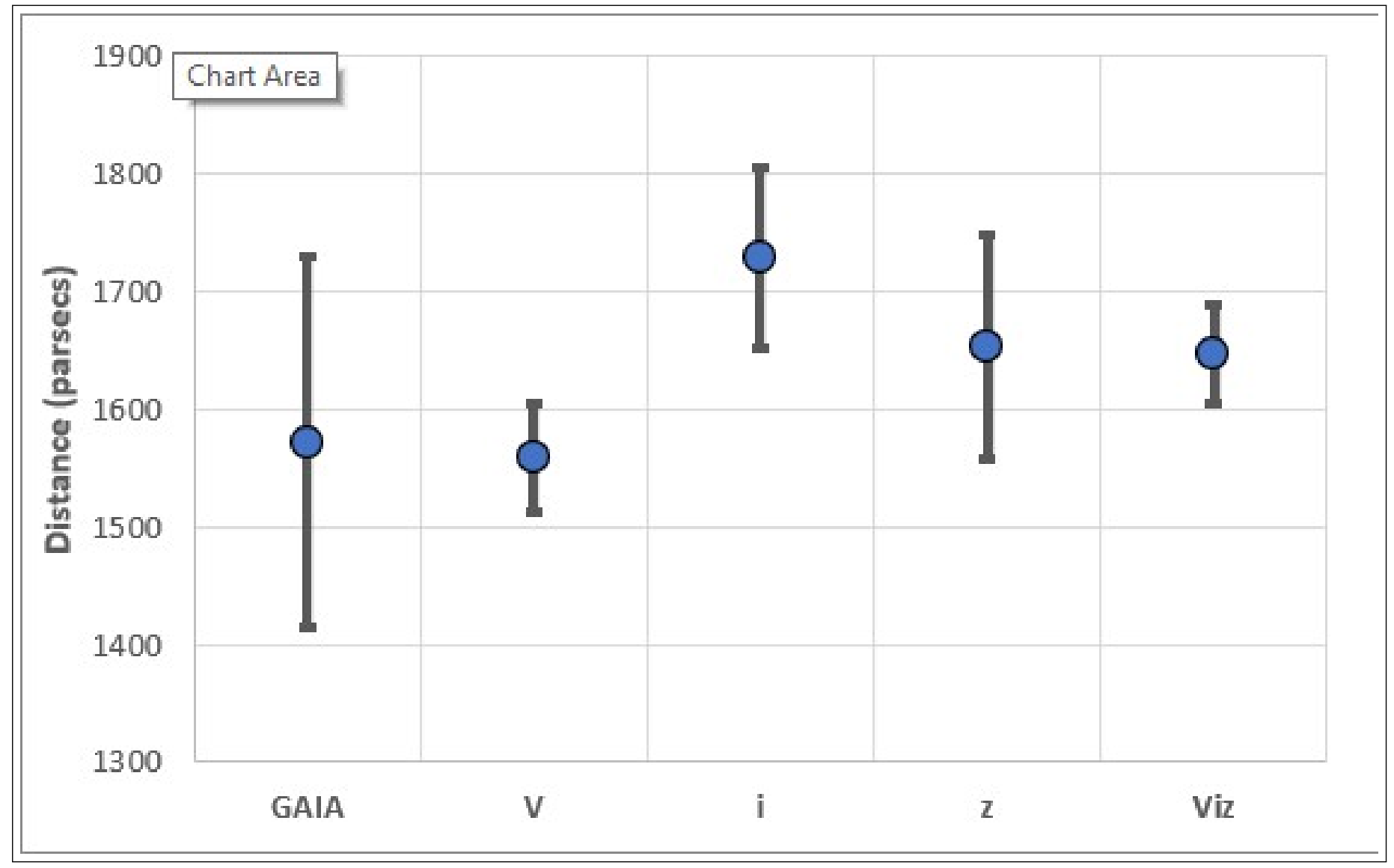

Fig. 9. Distance and Distance Errors 
Table 9. Measured Distance Variations for $D M$ And

\begin{tabular}{ccc}
\hline & Distance (parsec) & Distance Error (parsec) \\
\hline GAIA & 1572 & 157 \\
V & 1558 & 46 \\
i & 1728 & 77 \\
Zs & 1653 & 95 \\
Viz & 1647 & 42 \\
\hline
\end{tabular}

Figure 9 visually represents the distance measurements and distance errors. Figures 10 displays a comparison in magnitudes between DM And and another RR Lyrae star, AF Vel. The magnitude measurement for AF Vel strongly correlates with previously recorded GAIA data, so AF Vel was an ideal candidate when comparing DM And's magnitude to other RR Lyrae type stars. While DM And follows an expected path in the $\mathrm{B}$ and $\mathrm{V}$ filter there is a noticeable dimming of DM And's magnitude in the sdss-i and sdss-z filters compared to AF Vel before corrections noted in the discussion.

\section{DISCUSSION}

The twelve day observation period was not sufficient to witness a Blazhko Effect, as previous research has described. This lack of data does not discredit the possibility of a Blazhko Effect but could be explored in the future with longer-duration observations.

Initially, there was an unusually dim magnitude measured in the sdss-z filter of 12.4, 0.74 magnitudes dimmer than expected compared to AF Vel, and a smaller, but existing, deficiency of 0.1 in the sdss-i filter, with a magnitude of 11.8 , compared to the data from other filters. Astrosource was used to investigate the inconsistency. In Astrosource, the number of comparison stars used was changed to test whether there was a problem with the comparison stars and/or calibration or an actual effect from the star. In the first test, the first five comparison stars out of ten were used which resulted in a magnitude of 12.35 and after that the last five comparison stars were used which resulted in a magnitude 12.35 as well. To further investigate this, Astrosource was run ten times, using one of each of the ten comparison stars individually. The magnitudes of these ten tests were consistent with the results before varying in magni- tude from 12.35-12.45, which is not a substantial variation compared to the deficiency. This indicated the abnormality in the sdss-z filter was not caused by an outlier single comparison star or the calibration.

Further issues included that there was also a significant distance measurement variation between the measured distances of this study and previously measured distance done by GAIA. The range of the distance difference was 959 parsec between the lowest distance measurement done by GAIA at $1572 \pm 157$ parsec and the largest distance measurement in the sdss-z filter at $2531 \pm 160$ parsec.

However, it was discovered that the dimming in magnitude of the sdss-i and sdss-z filters and the variation in the distant measurements compared to GAIA was not an effect of DM And. In the PANSTARRS survey (Chambers et al., 2016) - used to calibrate the photometry for DM And, accessed via Aladin, there appears to be a spot in the center of some brighter stars, shown in Figure 12, where the pixels are black. When comparing the amount of light per pixel, to determine a stars magnitude and comparing it to DM And, they appear much dimmer than what they really are. Adjustments were made to the Astrosource program to avoid those stars contributing to the photometry. Once these issues were corrected for, the magnitude and distance values represented expected values for an RR Lyrae at the distance of DM And.

\section{CONCLUSION}

RR Lyrae stars are used to determine distance. The data collected in this study for DM And shows a typical trend for most RRab type RR Lyrae Pulsating stars, as shown above, with an average measured distance of $1647 \pm 42$ parsecs and an average period of 0.631 \pm 0.011 days. This is comparable to GAIA's measured distance of $1572 \pm 157 \mathrm{pc}$. The V filter derived distance, $1558 \pm 46 \mathrm{pc}$, individually agrees very well with the GAIA distance.

\section{ACKNOWLEDGMENTS}

This research has made use of the SIMBAD database, operated at CDS, Strasbourg, France 2000,A\&AS,143,9 , "The SIMBAD astronomical database", Wenger et al.

This research has made use of "Aladin sky atlas" developed at CDS, Strasbourg Observatory, France 2000A\&AS..143...33B and 2014ASPC..485..277B.

This work makes use of observations from the LCOGT network. 


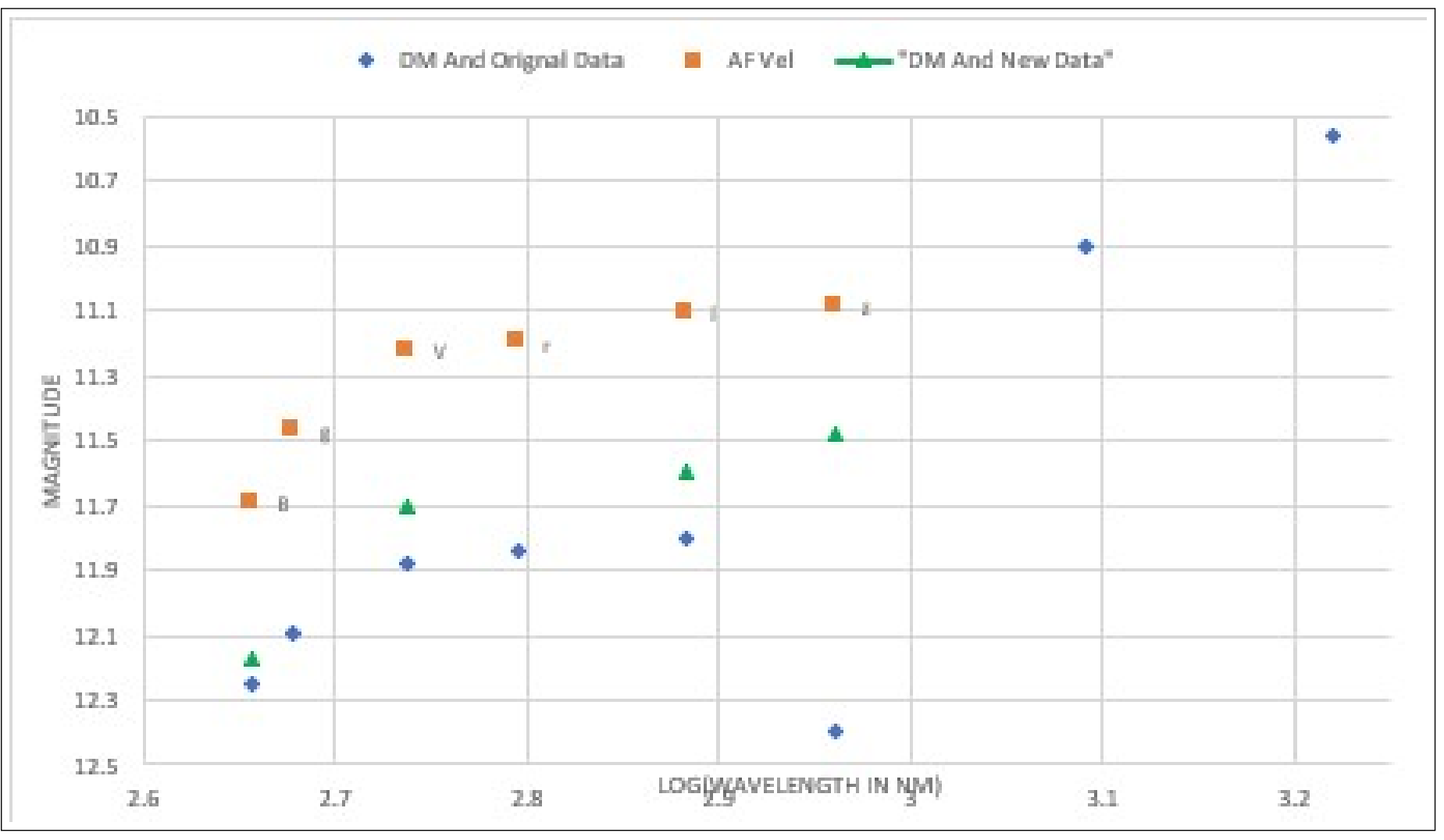

Fig. 10. Original magnitudes of DM And (both original magnitude measurements and current magnitude measurements) and AF Vel before any adjustments to m-M.

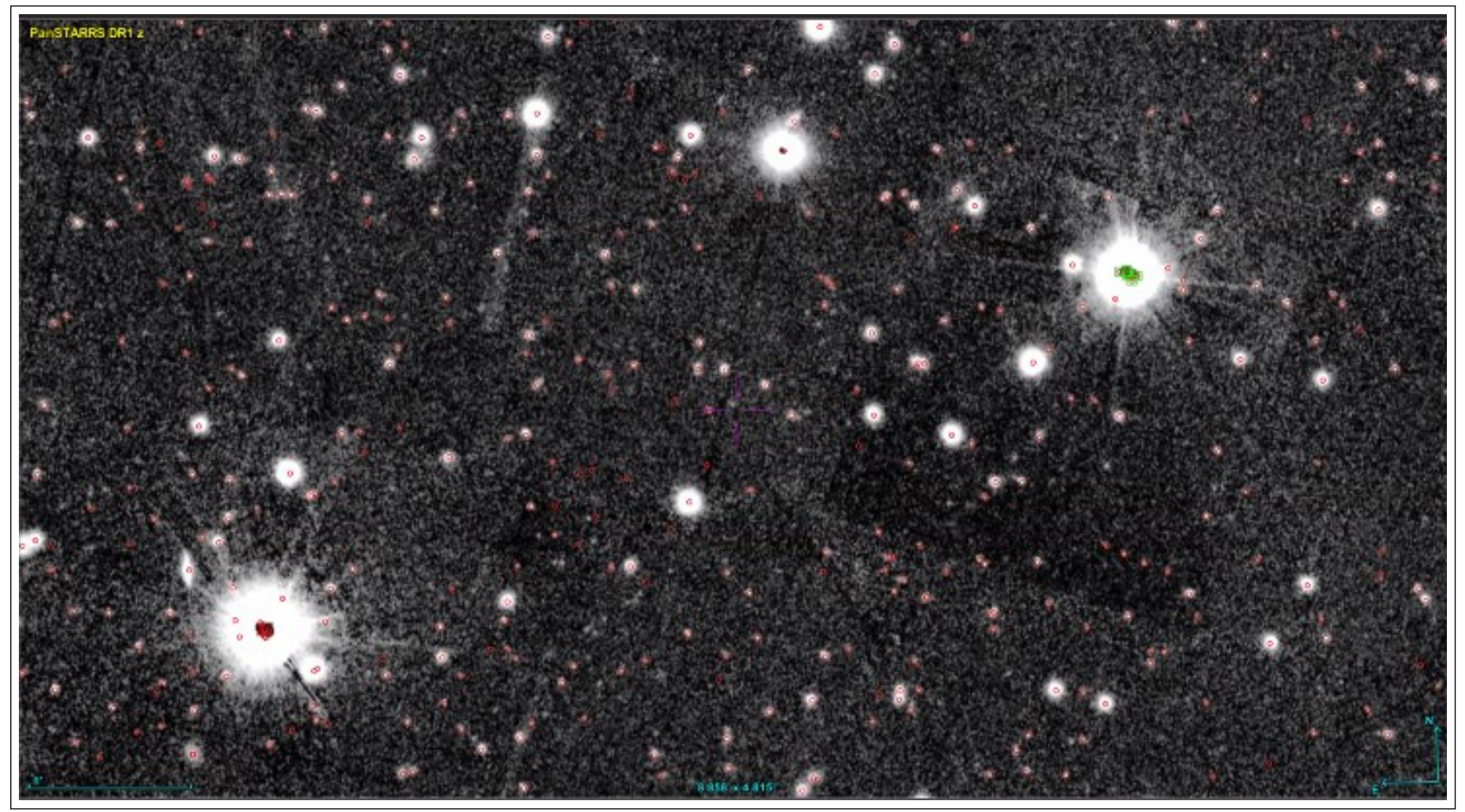

Fig. 11. The following is an image captured of the PANSTAARS survey in Aladin, showing the black spots in the center of the star, as well as multiple stars being counted in the black spot of a single star. 
This research has made use of the NASA/IPAC Infrared Science Archive, which is funded by the National Aeronautics and Space Administration and operated by the California Institute of Technology.

Special thanks to Michael Fitzgerald of Our Solar Siblings for his inspiration and generous and frequent support throughout this project.

\section{REFERENCES}

Altunin, I., Caputo, R., \& Tock, K. (2020). Period of eclipsing binary epic 201458798.

Beers, T. C., Chiba, M., Yoshii, Y., Platais, I., Hanson, R. B., Fuchs, B., \& Rossi, S. (2000). Kinematics of metal-poor stars in the galaxy. ii. proper motions for a large nonkinematically selected sample. The Astronomical Journal, 119(6), 2866.

Bertin, E. (2011). Automated morphometry with sextractor and psfex. In Astronomical data analysis software and systems $x x$ (Vol. 442, p. 435).

Bramich, D. M., Alsubai, K., Ferro, A. A., Parley, N., Cameron, A. C., Horne, K., ... West, R. (2014). Rr lyrae stars in the gcvs observed by the qatar exoplanet survey. arXiv preprint arXiv:1405.3417.

Brown, T., Baliber, N., Bianco, F., Bowman, M., Burleson, B., Conway, P., ... others (2013). Las cumbres observatory global telescope network. Publications of the Astronomical Society of the Pacific, 125(931), 1031.

Cáceres, C., \& Catelan, M. (2008). The periodluminosity relation of rr lyrae stars in the sdss photometric system. The Astrophysical Journal Supplement Series, 179(1), 242.

Catelan, M., Pritzl, B. J., \& Smith, H. A. (2004). The rr lyrae period-luminosity relation. i. theoretical calibration. The Astrophysical Journal Supplement Series, 154(2), 633.

Catelan, M., \& Smith, H. A. (2014). Pulsating stars. John Wiley \& Sons.

Chambers, K. C., Magnier, E., Metcalfe, N., Flewelling, H., Huber, M., Waters, C., ... others (2016). The pan-starrs1 surveys. arXiv preprint arXiv:1612.05560.

Fitzgerald, M. T. (2018). The our solar siblings pipeline: Tackling the data issues of the scaling problem for robotic telescope based astronomy education projects.

Fitzgerald, M. T., Gomez, E., Salimpour, S., Singleton, J., \& Wibowo, R. W. (2021). "astrosource": automating optical astronomy measurement, cali- bration and analysis for variable stellar sources from provided photometry. Journal of Open Source Software, 6(58), 2641.

Maintz, G. (2005). Proper identification of rr lyrae stars brighter than 12.5 mag. Astronomy \& Astrophysics, 442(1), 381-384.

Prusti, T., De Bruijne, J., Brown, A. G., Vallenari, A., Babusiaux, C., Bailer-Jones, C., ... others (2016). The gaia mission. Astronomy \& astrophysics, 595, A1.

Schlafly, E. F., \& Finkbeiner, D. P. (2011). Measuring reddening with sloan digital sky survey stellar spectra and recalibrating sfd. The Astrophysical Journal, 737(2), 103.

Schlegel, D. J., Finkbeiner, D. P., \& Davis, M. (1998). Maps of dust infrared emission for use in estimation of reddening and cosmic microwave background radiation foregrounds. The Astrophysical Journal, 500(2), 525.

Skarka, M. (2014). Bright blazhko rrab lyrae stars observed by asas and the superwasp surveys. Astronomy \& Astrophysics, 562, A90. 